\title{
À PROPOS DE QUELQUES PROCÉDÉS D'ACTUALISATION DES NOMS DE SENTIMENTS EN LETTON ET EN RUSSE
}

\author{
ELENA VLADIMIRSKA, JEL̨ENA GRIDINA
}

Université de Lettonie, Lettonie

\begin{abstract}
Résumé. Les noms des sentiments (p.ex. : amour, anxiété, etc.) ont un statut particulier. Ces noms n'ont pas de sous-catégories préétablies et sont récalcitrants à l'approximation : une sorte/forme d'amour est toujours de la amour. L'objet de la présente étude sont les Noms de sentiments (N_SENT) en letton et en russe, et les particularités de leur combinatoire. Alors que les études des N_SENT portent essentiellement sur l'analyse linguistique de leurs propriétés syntaxiques et sémantiques (Goosens, 2005; Mel'čuk, 1998; Tutin et Grossman, 2002), nous nous pencherons ici sur l'articulation entre les N_SENT et les unités appelées tantôt les noms 'métalinguistiques' (Flaux et Van de Velde, $2000: 26)$ tantôt les marqueurs discursifs (Paillard, 2011; Bui et al., 2017), à savoir čto-to tipa et čto-to vrode en russe et tips et tāds $k \bar{a}$ en letton. A travers la confrontation des langues appartenant à des groupes différents, nous chercherons à relever des régularités et des contraintes quant aux enjeux de l'actualisation des $\mathrm{N}$ de sentiments et des marqueurs dont ils constituent la portée.
\end{abstract}

Mots-clés : catégorisation, approximation, noms de sentiments, marqueurs discursifs

\section{INTRODUCTION}

L'objet de la présente étude sont les Noms de sentiments (N_SENT) en letton et en russe, et les particularités de leur combinatoire. Alors que les études des N_SENT portent essentiellement sur l'analyse linguistique de leurs propriétés syntaxiques et sémantiques (Goosens, 2005; Mel'čuk, 1998; Tutin et Grossman, 2002), nous nous pencherons ici sur l'articulation entre les N_SENT et les unités appelées tantôt les noms 'métalinguistiques' (Flaux et Van de Velde, $2000: 26$ ) tantôt les marqueurs discursifs (Paillard, 2011; Bui, Paillard et Vladimirska, 2017), à savoir čto-to tipa et čto-to vrode en russe et tips et tāds kā en letton.

Les domaines notionnels dont relèvent les N_SENT, ont quelques particularités notables. D’abord, ces $\mathrm{N}$ sont des $\mathrm{N}$ compacts (Culioli, 1999 : 14) c'està-dire non quantifiables, non fragmentables. Ils se manifestent à travers le $\mathrm{N}$ qui en est le support - actualisateur, tels que, par exemple, en français : une sorte de 
$N$, une espèce de $N$, en anglais : like, such as, etc. ; en russe čto-to/nečto vrode, etc. ; en letton : tāds $k \bar{a}$, it $k \bar{a}$, tipa, etc. Par ailleurs, les N_SENT renvoient à des états subjectifs/affectifs. Ces caractéristiques les rendent incompatibles avec la notion de conformité à une occurrence type - occurrence représentative objectivement repérable. La seule singularisation (fragmentation) possible est ainsi d'ordre qualitatif/subjectif ('ce que j'appelle $X$, un vrai $X$ pour moi...', ibid. : 14) qui s'effectue soit par un caractérisant qualitatif, comme adjectif, soit par un marqueur : un amour paternel, passionnel, etc.; une sorte d'amour, un genre d'amour, etc.

Parmi les actualisateurs des N_SENT il y a des unités lexicales relevant soit de la taxinomie, soit de la sémantique de comparaison et d'exemplification. Il est à noter que les premiers sont dominants dans notre corpus russe : čto-to vrode (quelque chose dans le genre de), čto-to tipa (quelque chose du type de...). Ces marqueurs ont fait l'objet de multiples études, parmi lesquelles Sakhno, 2002, 2017; Lapteva, 1983; Bogdanova-Beglaryan, 2014; Savchenko 2015. Alors que les seconds sont propres au letton : tā $d s k \bar{a}$ (tel comme), it kāa (comme si) (pour le marqueur it $k \bar{a}$ voir A. Kalnača (2010)). Par ailleurs, čto-to tipa, est très rare devant les N_SENT en russe, tandis que čto-to vrode les précède avec une fréquence remarquable.

Ces observations nous ont conduites à nous interroger sur les propriétés sémantiques des N_SENT et celles des marqueurs qui les précèdent. Dans ce qui suit, nous procéderons à une analyse des exemples tirés du corpus russe et letton afin de proposer une explication de certaines contraintes liées à l'association des N_SENT aux marqueurs qui en sont les actualisateurs.

\section{1 ČTO-TO TIPA}

Tipa et vrode appartiennent, selon la classification proposée par Paillard (2017), à la classe des mots du discours (MD) écran - la notion d'écran renvoyant à la fois à ce qui cache (faire écran) et à ce sur quoi on projette - et relèvent de la problématique du dire : on n'a pas les mots adéquats pour dire l'état de choses $\mathrm{R}$.

Bien que le MD tipa soit très répandu en russe - surtout en russe oral, où la fréquence de son usage en fait parfois un tic verbal - dans notre corpus, la locution čto-to tipa est particulièrement rare devant N_SENT. En effet, tipa relève du discernement (catégorisation en référence à un type (Culioli, 1999 : 11-12) ce qui le rend compatible avec les N-événement (il n'y a aucun problème de dire, par exemple, on pročel čto-to tipa lekcii//litt. : il a fait quelque chose 'tipa' conférence) mais crée une contrainte lorsqu'il s'agit des N_SENT (on ispytyval čto-to tipa gor'a/l'ubvi, etc.//il éprouvait quelque chose 'tipa' chagrin/amour). Avec le type, la notion est première et sert à catégoriser/formater ce que l'on a (l'état de choses R). Sémantiquement, type vient de pièce, portant une empreinte, servant à reproduire des empreintes identiques, ou encore, de moule, modèle idéal qui détermine la forme d'une série d'objets qui en dérivent (TLF, En ligne 1). Cette dynamique entre en contradiction avec la sémantique des N_SENT : renvoyant aux états subjectifs ils résistent à tout ce qui est de l'ordre du formatage 
et adoptent un mouvement inverse où l'on part de 'ce que l'on a' - aussi vague et incompréhensible qu'il soit - et on cherche la notion dont il est l'actualisation. Les rares exemples avec čto-to tipa $+N$ _SENT ne font que confirmer cette hypothèse :

Ex.1. U neye s proshlym yeye muzhem bylo chto-to ser'yeznoye, skazal Slava. - Chto-to tipa lyubvi. V takikh situatsiyam plokho byt' vtorym. (En ligne 2) - Il y avait quelque chose de sérieux entre elle et son ex-mari, - dit Slava. - Quelque chose 'tipa' amour. Dans telles situations, c'est mal d'être le deuxième. - traduit par les auteurs de l'article.

Ex.2. Yesli by prostyye i sil'no p'yushchiye lyudi, kotoryye rabotali bok o bok s Bilyatdinom $\mathrm{v}$ odnom tsekhu pod rukovodstvom Goremykinoy, ili, kak zvalas' ona dlya kratkosti, Andrevny, yesli by oni mogli obratit'sya myslyami k vysokim primeram, oni by, ne isklyucheno, ispytali (vmesto styda i zameshatel'stva) chto-to tipa professional'noy gordosti. (En ligne 2) - Et si des gens simples, de gros buveurs qui travaillaient côte à côte avec Bilyaddin dans le même atelier sous la direction de Goremykina, ou, comme elle s'appelait en abrégé, Andrevna, s'ils pouvaient diriger leurs pensées vers les hauts exemples, ils auraient éprouvé (au lieu de la honte et de la confusion) quelque chose 'tipa' fierté professionnelle. - traduit par les auteurs de l'article.

Dans les exemples ci-dessus, la compatibilité du N_SENT avec tipa est frayée par le contexte gauche. Ainsi, dans l'ex.1 : čto-to ser'eznoe//quelque chose de sérieux construit une occurrence, alors que tipa ljubvi//'tipa' amour nomme l'occurrence en question. Par ailleurs, l'énonciateur n'est pas ici un expérienceur du sentiment d'amour - il s'agit d'un sentiment éprouvé par la femme qui l'intéresse, pour son ex-mari : le formatage qu'effectue tipa affecte la prise en charge énonciative et permet de construire une position S0 distancié.

Dans l'ex.2 : čto-to tipa relève du contraste entre différents $\mathrm{N}$ la honte et de la confusion vs fierté professionnelle, et du problème de catégorisation : quel est le bon $\mathrm{N}$ pour R. Opposé à la honte et la confusion du contexte gauche (le sentiment réellement éprouvé par les ouvriers), fierté professionnelle a ici une coloration nettement ironique. Par ailleurs, il s'agit d'une formation discursive stéréotypé, renvoyant à l'hypocrisie d'un discours officiel, contrairement à la honte et la confusion lesquelles sont de 'vrais' sentiments expérimentés subjectivement.

Quant au letton, les noms relevant de la taxinomie ne constituent pas les supports actualisant les N_SENT. On ne les retrouve que dans les contextes purement taxinomiques tels que :

Ex.3 Ir vairāki mīlestības tipi, kuriem mēs izejam cauri dzīves laikā. (En ligne 3) - Il y a de plusieurs types d'amour par lesquels nous passons durant la vie. 
Ainsi, étant donné deux mouvements inverses - celui de la notion vers les occurrences de la notion (sémantique véhiculée par 'tipa') d'une part, et, d'autre part, celui de l'individu vers la notion dont il est une manifestation singulière - les N_SENT et des états émotionnels s'associent plutôt aux marqueurs relevant de la seconde dynamique et permettant ainsi une singularisation d'ordre qualitatif. Tels sont čto-to vrode pour le russe et tāds kā pour le letton.

\section{2 ČTO-TO VRODE}

Relevant sémantiquement de 'rod' (genre), le MD vrode renvoie à l'idée des origines communes lesquelles conditionnent une certaine ressemblance, tout en maintenant la différenciation au cœur de sa sémantique : la composante diachronique/évolution/changements fait en sorte que tout en ayant les mêmes origines, les entités constituent un paradigme ouvert à des variations. Si dans le cas de tipa on est dans l'optique de formatage (notion $=>$ occurrence), ici, au contraire, on part de $\mathrm{R}$ en cherchant à le 'capter' par les mots, lui donner une visibilité, un mode de présence. Autrement dit, avec čto-to vrode, $\mathrm{p}$ (le dire) ne dit $\mathrm{R}$ que partiellement, le contexte montrant souvent plusieurs 'tentatives' de rendre compte de R.

\section{Ex.4. On ispytyval tol'ko chto-to vrode oshchushcheniya koshmara, strashnoy neleposti togo, chto proiskhodit, sna, kotoryy on ne $\mathrm{v}$ silakh prervat'. "Kak khorosho togda bylo u morya", - vdrug ostro i bystro podumalos' yemu, - "a teper' vot...” (En ligne 2) - Il ne ressentait qu'une sorte de 'čto-to vrode' sensation de cauchemar, de terrible absurdité de ce qui se passait, d'un rêve qu'il ne pouvait pas interrompre. "Comme c'était bon alors au bord de la mer", pensa-t-il soudainement et vivement, "et maintenant voilà..." - traduit par les auteurs de l'article. Pour faciliter la lecture, on a choisi de traduire le marqueur čto-to vrode par le marquer français une sorte de.}

Ainsi, dans l'ex.4 ci-dessus, on part de ce que l'on a - une sensation - et on actualise le N_SENT :-cauchemar, terrible absurdité, rêve-aucune de ces notions ne prétend dire $\mathrm{R}$ pleinement, celui-ci étant posé comme fondamentalement indicible. On observe la même dynamique dans l'ex.5 où la notion même de sensation n'est pas stabilisée en tant que nom (p) de R:

Ex.5 Pervyy moment - eto chto-to vrode oshchushcheniya, po krayney mere, $\mathrm{v}$ nem preobladayet oshchushcheniye; tosklivoye, ono pokhozhe na tot obraz, kotoryy Aratov uvidel v stereoskope. (En ligne 2) - Le premier moment c'est une sorte de 'čto-to vrode' sensation, du moins la sensation y prévaut; triste, elle ressemble à l'image que Aratov a vue dans le stéréoscope. - traduit par les auteurs de l'article.

Lorsque l'hyperonyme (sensation de/sentiment de) n'est pas actualisé, le cotexte droit, relevant de l'ordre de l'observable, vise à expliciter le mode singulier d'actualisation du $\mathrm{N}$ sentiment : 
Ex.6. No, kogda ya uvidela, kak mashina pereyekhala sestru, so mnoy sluchilos', navernoye, chto-to vrode pomeshatel'stva. Ya perestala zasypat' $v$ temnote, vso vremya videla yeyo litso... (En ligne 2) - Mais quand j'ai vu la voiture renverser ma sœur, j'ai été prise d'une sorte de 'čto-to vrode' folie. J'ai cessé de m'endormir dans le noir, tout le temps j'ai vu son visage ... - traduit par les auteurs de l'article.

Ex.7. Buduchi slabym i truslivym, seychas on vdrug pochuvstvoval dazhe chto-to vrode gordosti: on, kak ravnyy, beseduyet s lyud'mi iz ugro! Bylo pokhozhe na stsenu iz video. (En ligne 2) - Étant de nature faible et lâche, il a soudainement ressenti même une sorte d' 'čto-to vrode' orgueil: lui, en tant qu'égal, parle aux gens de la brigade criminelle ! C'était comme une scène d'un film. - traduit par les auteurs de l'article.

Ainsi, ce sont les symptômes décrits dans le cotexte droit de l'ex.6 - J'ai cessé de m'endormir dans le noir, tout le temps j'ai vu son visage - qui justifient, dans un certain degré, le choix du N (folie). De même, dans l'ex.7, le contexte droit : lui, en tant qu'égal, parle aux gens de la brigade criminelle - conditionne la sélection de $\mathrm{p}$ (orgueil).

\section{TĀDS KĀ}

Comme nous l'avons mentionné plus haut, les marqueurs 'taxinomiques' ne participent pas à l'actualisation des N_SENT en letton: klase (classe), kärta (ordre), suga (espèce) etc. ne quittent pratiquement jamais le champ du domaine scientifique ou technique. Employés dans d'autres contextes, ils véhiculent néanmoins leur sémantique de catégorisation. Lorsqu'il s'agit de la sémantique d'approximation ou de la problématique de nommer $\mathrm{R}$, le letton a recours aux unités lexicales relevant de la sémantique de comparaison (ex.8) ou exemplification (ex.9). Dans ces exemples tā $d s k \bar{a}$ marque un rapprochement, selon un rapport d'equivalence ou d'exemplification, de deux unités nominales : $\mathrm{X}$ est tāds kā (tel que/comme) Y.

Ex.8. Rìgas Dzīve bija tāds kā pilsētas termometrs - tur vienmēr tika publicētas aktuālākās pilsētas leǵendas, intervijas ar skandalozākajām personām un vissmagākās filosofiskās pārdomas par dzìves jēgu... (En ligne 4) - Rigas Dzive était comme thermomètre de la ville - là on publiait des anecdotes les plus récentes de la ville, des interviews des personnes les plus scandaleuses et des réflexions philosophiques les plus tordues sur le sens de la vie...- traduit par les auteurs de l'article.

Ex.9. Šobrīd procesā iesaistītajiem dalībniekiem - gan tiesnesim, gan prokuroram, gan advokātam, gan apsūdzētajiem, gan cietušajiem Par visiem kopā ir vairāk nekā 15 legālu iemeslu, lai lūgtu atlikt tiesu, sākot ar tādiem kā slimība, atvaļinājums, mācības, aizṇemtība citā lietā. 
(En ligne 4) - À l'heure actuelle, pour toutes les parties impliquées aux procès - juge, procureur, avocat, accusés et victimes, il existe plus de 15 raisons légitimes pour rapporter le procès, à commencer par telles que maladie, congé, études, implication aux aurtes cas. - traduit par les auteurs de l'article.

Le journal Rigas Dzìve de l'ex.8 (X) - est comparé au thermomètre de la ville $(\mathrm{Y})$; les 'raisons légitimes' de l'ex.9 (X) sont explicitées/énumérées dans la partie droite (Y) introduite par tāds $k \bar{a}$.

Lorsque la problématique qui est en jeu consiste en comment dire $R$ et, notamment, comment rendre compte d'un état émotionnel, comment l'expliciter par le biais de la notion, nous observons deux changement formels de l'énoncé : a) l'omission de l'élément $\mathrm{X}$ et b) l'accord en genre et en nombre de tāds $k \bar{a}$ ne se fait plus avec l'élément $\mathrm{X}$ du contexte gauche, comme dans le cas de l'exemplification, mais avec l'élément constituant la portée de tāds kā dans le contexte droit. (ex.10, 11).

Ex.10 Nemāku izteikt to sajūtu. Jā, nerealitāte. Tāda kā sapña sajūta, kad gribi un nevari atmosties. Atcerējos - tāda sajūta kā jaunībā, kad esi nonācis kino filmas iekšienē. (En ligne 4) - Je ne peux pas exprimer cette sensation. Oui, irréalité. Comme une sensation de rêve quand tu veux et que tu ne peux pas te réveiller. Je me suis souvenu un sentiment comme dans la jeunesse, quand tu entres à l'intérier d'un film. - traduit par les auteurs de l'article.

Ex.11. Tomēr, atšķirībā no draudzības ar Henriju, es no Ābrama esmu jutis tādu kā vēsumu, īsta siltuma un sirsnības trūkumu, allaž zināmu distanci un atturību. (En ligne 4) - Cependant, contrairement à mon amitié avec Henry, j'ai senti d'Abraham, comme une froideur, un manque de chaleur et de sincérité, une distance et une abstinence. traduit par les auteurs de l'article.

On note ici la même dynamique que dans les exemples du russe. Dans l'ex.10, un échec de la mise en mots explicitement avoué dans le contexte gauche : Je ne peux pas exprimer cette sensation - est suivi de plusieurs tentatives de dire $\mathrm{R}$ irréalité, une sensation de rêve, quand tu veux et que tu ne peux pas te réveiller. De même, dans l'ex.11 : une fois le N_SENT actualisé par tāds $k \bar{a}$, d'autres dires sont là pour qualifier/individuer ce qui est le cas : froideur, un manque de chaleur et de sincérité, une distance et une abstinence. Ainsi, à partir de sa sémantique de base qui est celle de comparaison, tāds $k \bar{a}$ construit un rapport $\mathrm{p}-\mathrm{R}$ non stabilisé, voire brouillé, en effectuant par ceci une ouverture vers d'autre façon de dire $\mathrm{R}$.

\section{CONCLUSION}

La sémantique spécifique de N_SENT influence leur combinatoire, et notamment les mots-actualisateurs qui y sont associés. A partir de leur sémantique propre, 
les mots-supports permettent de construire une dynamique d'individuation (QLT), en partant de 'ce qui est', et en cherchant la notion - N_SENT - dont il est l'actualisation. Dans cette entreprise, le letton et le russe ont recours aux supports sémantiques différents mais lesquels effectuent - chacun à sa façon - la réalisation de la sémantique de singularisation. Dans le cas du letton, on part de la base de la comparaison ( $t \bar{a} d s k \bar{a})$, alors que dans le cas du russe, on s'appuie sur un marqueur vrode d'origine sémantique ambiguë - catégorisant/individuant - avec cependant une pondération sur la seconde composante. Une étude approfondie du combinatoire des N_SENT et de l'interaction entre leur sémantique et celle des marqueurs dans une perspective interlangue, incluant des langues romanes, fera objet des publications postérieures des auteures.

\section{BIBLIOGRAPHIE}

Bogdanova-Beglaryan, N. V. (2014) Diskursivnaya yedinitsa tipa (togo chto): funktsionirovaniye v ustnoy spontannoy rechi i vozmozhnosti leksikograficheskogo opisaniya. Problemy istorii, filologii, kul'tury, 3(45) : 252-255.

Bui, T. H. A., Paillard, D. et Vladimirska, E. (2017) Étude de certains marqueurs discursifs 'vrai' en français, khmer, russe et vietnamien. Langages, (3) : 33-48.

Culioli, A. (1999) Pour une linguistique de l'énonciation. Tome 3. Paris : Ophrys.

Flaux, N. et Van de Velde, D. (2000) Les noms en français : esquisse de classement. Parsi : Ophrys.

Goossens, V. (2005) Les noms de sentiment. Esquisse de typologie sémantique fondée sur les collocations verbales. Lidil. Revue de linguistique et de didactique des langues, (32) : 103-121.

Kalnača, A. (2010) Partikula IT KĀ un modalitāte. Latvistika un somugristika Latvijas Universitātē, (p.39-49). Latvijas Universitāte.

Lapteva, O. A. (1983) Tipa ili vrode. Voprosy yazykoznaniya, (1) : 39-51.

Mel'čuk, I. (1998) Collocations and lexical functions. In A.P. Cowie (ed.) Phraseology. Theory, analysis, and applications (p. 23-53). Oxford: Clarendon Press.

Paillard, D. (2011) Marqueurs discursifs et scène énonciative. In S. Hancil (dir.) Marqueurs discursifs et subjectivité (p. 11-32). Presses universitaires de Rouen et du Havre.

Paillard, D. (2017) Scène énonciative et types de marqueurs discursifs. Langages, (3) : 17-32.

Sakhno, S. (2002) O slove vrode: prototipichnoct' 'za neimeniyem luchshego'. Rusistika segodnya, 1-4: 45-57.

Sakhno, S. (2017) Polyfonctionnalité et transcategorialité des morphèmes russes vrode et tipa. Mots de liaison et d'intégration: Prépositions, conjonctions et connecteurs, 34 : 197-213.

Savchenko, D. S. (2015) On possible functions of xind vrode/tipa/napodobie 'like' y in Russian colloquial speech. Kommunikativnyye issledovaniya, (4) : 100-108.

Tutin, A. et Grossmann, F. (2002) Collocations régulières et irrégulières : esquisse de typologie du phénomène collocatif. Revue française de linguistique appliquée, 7(1) : 7-25.

Vassiliadou, H., Vladimirska E., Gridina, J., Turlā, D., Lammert, M., Benninger, C., GerhardKrait, F. (à paraître) Clear vs approximate categorization in French and Latvian. Book Series by De Gruyter Mouton.

\section{SITOGRAPHIE}

1) [En ligne 1] Trésor de la langue française (TLF). Disponible en ligne http://atilf.atilf.fr/ [Consulté le 28 juin 2019].

2) [En ligne 2] Natsional'nyy korpus russkogo yazyka. Disponible en ligne http://www. ruscorpora.ru/new/ [Consulté le 28 juin 2019]. 
3) [En ligne 3] Ginta Filia Solis. Disponible en ligne https://gintafiliasolis.wordpress.com/ [Consulté le 28 juin 2019].

4) [En ligne 4] IvTenTen Latvian Corpus form the Web. Disponible en ligne http://nosketch. korpuss.lv/run.cgi/first_form [Consulté le 28 juin 2019].

\title{
ABOUT SOME PROCEDURES OF ACTUALIZATION OF THE NAMES OF FEELINGS IN LATVIAN AND RUSSIAN
}

\begin{abstract}
The names of feelings (e.g. love, anxiety, etc.) have a particular status: they have no pre-established subcategories and, in this sense, resist the approximation: kind/form of love is still love. The subject of the present study are the names of feelings (N_SENT) in Latvian and Russian, and the peculiarities of their combinatorics. Whereas the majority of N_SENT studies focus on the linguistic analysis of their syntactic and semantic properties (e.g. Goosens, 2005; Mel'čuk, 1998; Tutin and Grossman, 2002), we focus on units sometimes referred to as 'metalinguistic' words (Flaux and Van de Velde, 2000: 26) or 'discourse markers' (Paillard, 2011; Bui et al., 2017) which precede the N_SENT (e.g. čto-to vrode / kind of; tāds kā / like). By contrasting the languages belonging to different groups, we will seek to identify regularities and constraints related to the connection of N_SENT to markers which enable their actualization.
\end{abstract}

Key words: categorization, approximation, names of feelings, discourse markers

Elena Vladimirska (Dr.Philol., professeure à l'Université de Lettonie) titulaire d'un doctorat en sciences du langage de l'Université Paris 3 Sorbonne Nouvelle, elle dirige le Département $\mathrm{d}>$ études romanes à $1>$ Université de Lettonie et le programme de Master de Langues et cultures romanes. Ses travaux de recherches portent sur la sémantique, l'énonciation, la prosodie, ainsi que sur les études contrastives de l’oral. Courriel : jelena.vladimirska@lu.lv

Jel̦ena Gridina (Dr.Philol., maître de conférences à l'Université de Lettonie) titulaire d'un doctorat en linguistique appliquée soutenu à l'Université de Lettonie, elle enseigne les langues romanes au département d'études romanes de l'Université de Lettonie. Ses principaux domaines de recherche concernent la didactique des langues romanes, le plurilinguisme et l'intercompréhension. Courriel : jelena.gridina@lu.lv 